\title{
Studies on Novel Synthetic Methodologies, Part XII: An Efficient One-Pot Access to 6,6a-Dihydroisoindolo[2,1-a]quinazoline-5,11-diones and 5-Phenylisoindolo[2,1-a]quinazolin-11(6aH)-ones
}

\author{
Koneni V. Sashidhara, ${ }^{* a}$ Gopala Reddy Palnati, ${ }^{a}$ Ranga Prasad Dodda, ${ }^{a}$ Srinivasa Rao Avula, ${ }^{\text {a }}$ Priyanka Swami ${ }^{\mathrm{b}}$ \\ a Medicinal and Process Chemistry Division, Central Drug Research Institute, CSIR-CDRI, Lucknow 226001, India \\ Fax+91(522)2623405; E-mail: sashidhar123@gmail.com; E-mail: kv_sashidhara@cdri.res.in \\ ${ }^{b}$ Medicinal Chemistry, National Institute of Pharmaceutical Education and Research (NIPER), Raebareli 229010, India \\ Received: 26.10.2012; Accepted after revision: 15.11.2012
}

\begin{abstract}
A simple and efficient procedure for the construction of 6,6a-dihydroisoindolo[2,1-a]quinazoline-5,11-dione and 5-phenylisoindolo[2,1-a]quinazolin-11(6aH)-one derivatives in acetic acid under catalyst-free conditions is described. Attractive features of this methodology are its versatility, ready availability of starting materials and the efficiency in creating a complex core in a single operation.
\end{abstract}

Key words: one-pot synthesis, catalyst-free, 6,6a-dihydroisoindolo $[2,1-a]$ quinazoline-5,11-dione, 5-phenylisoindolo[2,1-a]quinazolin-11(6a $H)$-one, 2-formyl benzoic acid

Quinazolines, quinazolinones and isoindolinone, represent common structural units in many natural alkaloids including rutaecarpine (1). They also show a range of biological activities. ${ }^{1}$ In addition many unnatural quinazolinones show significant biological activities acting as anticancer, ${ }^{2}$ antibacterial, ${ }^{3}$ anti-inflammatory, ${ }^{4}$ antihypertensive, ${ }^{5}$ antidiabetic, ${ }^{6}$ anticonvulsant, ${ }^{7}$ and antianxietic agents. ${ }^{8}$ Figure 1 shows the chemical structures of some potent biologically important quinazolinone, isoindolin1-one-based compounds and the general structure of our synthesized prototype. The kinesin spindle protein inhibitor $2^{9}$ displays antimitotic activity and is in phase II clinical trials, while methaqualone (3) is a clinically used sedative. ${ }^{10}$ Furthermore, diproqualone $\mathbf{4}$ is used primarily for the treatment of inflammatory pain associated with osteoarthritis, ${ }^{11}$ while compound $\mathbf{5}$ possesses potent nicotinic acid receptor agonist activity for the treatment of dyslipidemia. ${ }^{12}$ The isoindolinone (phthalimidine) motif occurs as a core structure not only in several natural products such as lennoxamine $(6)^{13}$ but also in a number of pharmacologically relevant synthetic molecules, such as pagoclone $(7) .{ }^{14}$
Hybrid molecules that combine two heterocyclic structural units of different nature generally lend themselves well to rational drug design and often possess improved biological activities. ${ }^{15} \mathrm{~A}$ number of biologically potent hybrids have been described in the literature such as steroid antibiotics, ${ }^{16}$ steroid nucleosides, ${ }^{17}$ triterpenoid peptides, ${ }^{18}$ and DNA-cleaving-agent amino acids. ${ }^{19}$ Multicomponent reactions (MCRs), have proven to be one of the most powerful methods to access complex structures in a single synthetic operation from simple building blocks ${ }^{20}$ and are very useful in the construction of diverse chemical libraries of 'drug-like' molecules. ${ }^{21,22}$ Recently, Pal et al. synthesized 6,6a-dihydroisoindolo[2,1-a]quinazoline-5,11-dione derivatives 8 by employing a threecomponent reaction of isatoic anhydride, an amine and 2formylbenzoic acid, using montmorillonite $\mathrm{K} 10$ as the catalyst. ${ }^{23 a}$ Additionally, Bunce et al. reported the synthesis of substituted quinazolinones using a dissolving metal reduction-condensative cyclization strategy. ${ }^{23 b}$ However, such syntheses are still suboptimal because of use of catalysts, forcing reaction conditions and long reaction times.

As a part of our ongoing studies devoted towards the development of a practical synthesis of biologically interesting heterocyclic molecules, ${ }^{24}$ we have explored the possibility of an operatively simple, catalyst-free synthesis of 6,6a-dihydroisoindolo[2,1-a]quinazoline-5,11-dione derivatives. In the initial phase of this study we investigated the cyclization reaction of 2-formylbenzoic acid (3) with isatoic anhydride (1) and $p$-toluidine (2i) in the absence of catalyst in ethanol under heating (Scheme 1) for two hours which yielded the required compound $4 \mathbf{i}$ in $45 \%$ yield (Table 1 , entry 1 ). Further optimization re-

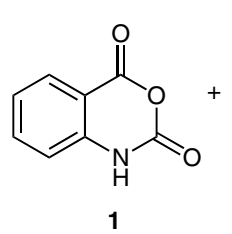<smiles>Cc1ccc(N)cc1</smiles><smiles>O=Cc1ccccc1C(=O)O</smiles><smiles>Cc1ccc(N2C(=O)c3ccccc3N3C(=O)c4ccccc4C23)cc1</smiles>

$4 \mathbf{i}$

Scheme 1 Reaction of isatoic anhydride (1), p-toluidine (2i) and 2-formylbenzoic acid (3)

SYNLETT 2013, 24, 0105-0113

Advanced online publication: 11.12.2012

DOI: 10.1055/s-0032-1317761; Art ID: ST-2012-D0922-L

(C) Georg Thieme Verlag Stuttgart · New York 


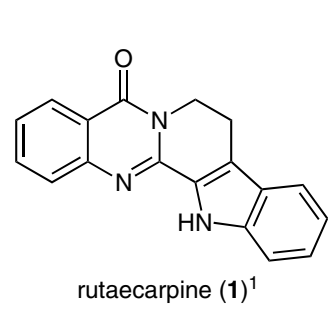<smiles>CCC(c1nc2ccccc2c(=O)n1Cc1ccccc1)N(CCCNC)C(=O)c1ccc(Br)cc1</smiles><smiles>Cc1ccccc1-n1c(C)nc2ccccc2c1=O</smiles>

methaqualone $(\mathbf{3})^{10}$<smiles>Cc1nc2ccccc2c(=O)n1CC(O)CO</smiles><smiles>O=C1C=C(CCCC2CC2)C2C(=O)NC(C(F)F)=NC2O1</smiles><smiles>COc1ccc2c(c1OC)C(=O)N1CCc3cc4c(cc3CC21)OCO4</smiles>

lennoxamine $(6)^{13}$<smiles>CC(C)CCC(=O)CC1c2ccccc2C(=O)N1c1ccc2ccc(Cl)nc2n1</smiles><smiles>O=C1c2ccccc2N2NC1c1ccccc1C2=O</smiles>

our prototype (8)

pagoclone $(7)^{14}$

Figure 1 Biologically active quinazolinone, isoindolinone-based compounds $1-7$ and our prototype 8

sults are presented in Table 1. Replacement of ethanol with acetic acid caused a sharp increase in the yield of this reaction ( $85 \%$; Table 1 , entry 6 ). The solvent was found to have a dramatic impact on the efficiency of the reaction (Table 1, entries 9-13). On the other hand, the use of $\mathrm{CF}_{3} \mathrm{COOH}$ completely closed down the reaction (Table 1, entry 8). The cyclization ability of various binary solvent systems such as ethanol-acetic acid (Table 1, entry 9), acetonitrile-acetic acid (Table 1, entry 10) and DMFacetic acid (Table 1, entry 11) was also tried without success.

We also carried out the reaction in the absence of acetic acid to confirm the cyclization ability of acetic acid. Thus, among the solvents screened, only the acetic acid promoted effective cyclization in high yield and short reaction time (Table 1, entry 13). Further increases in the reaction time did not increase the yield of the reaction (Table 1, entries 6 and 12).

The scope of this one-pot three-component synthesis of 6,6a-dihydroisoindolo[2,1-a]quinazoline-5,11-dione derivatives was next investigated. To explore the applicability of our reaction, we employed a variety of substituted aliphatic and aromatic amine derivatives in the reaction. Though the reaction worked well in all cases, the yields were better for the more nucleophilic aliphatic amines than aromatic amines (Table 2).
Table 1 Effect of Reaction Conditions on the Cyclization of Isatoic Anhydride (1), $p$-Toluidine (2i) and 2-Formylbenzoic Acid (3)

\begin{tabular}{clcll}
\hline Entry & Solvent & Temp $\left({ }^{\circ} \mathrm{C}\right)$ & Time (h) & Yield $(\%)^{\mathrm{a}}$ \\
\hline 1 & EtOH & 90 & 2.0 & 45 \\
2 & i-PrOH & 90 & 2.0 & 35 \\
3 & DMF & 110 & 2.0 & 30 \\
4 & DMSO & 110 & 2.0 & 30 \\
5 & MeCN & 90 & 2.0 & 40 \\
6 & AcOH & 110 & 2.0 & 85 \\
7 & $1,4-$ dioxane & 110 & 2.0 & 40 \\
8 & CF ${ }_{3} \mathrm{COOH}$ & 110 & 2.0 & - \\
9 & AcOH-EtOH (7:3) & 100 & 2.0 & 70 \\
10 & AcOH-MeCN (7:3) & 100 & 2.0 & 73 \\
11 & AcOH-DMF (7:3) & 110 & 2.0 & 70 \\
12 & AcOH & 110 & 1.0 & 85 \\
13 & AcOH & 110 & 0.75 & 85 \\
14 & AcONa & 90 & 2.0 & 10 \\
\hline
\end{tabular}

${ }^{a}$ Yields after recrystallization from chloroform-hexane (2:8). 
All the synthesized ${ }^{25}$ compounds were confirmed by ${ }^{1} \mathrm{H}$ NMR, ${ }^{13} \mathrm{C}$ NMR, IR spectroscopy and mass spectrometry and their comparison with published data in the case of known compounds $(\mathbf{4 a}-\mathbf{e}, \mathbf{h}, \mathbf{m}, \mathbf{n}){ }^{23}$

A plausible mechanism for the formation of $6,6 \mathrm{a}$-dihydroisoindolo[2,1-a]quinazoline-5,11-diones is depicted in Scheme 2. ${ }^{23 a}$ In order to elucidate the possible role of acetic acid the reaction was performed at two different $\mathrm{p} K_{\mathrm{a}}$ values. The reaction with sodium acetate (a weak base) in ethanol (Table 1, entry 14) resulted in low yield of the product. The best results were obtained in acetic acid ( $\mathrm{p} K_{\mathrm{a}} 4.76$; Table 1, entries 6, 12 and 13), while the reaction when performed in trifluoroacetic acid $\left(\mathrm{p} K_{\mathrm{a}} 0.23\right.$; Table 1, entry 8) did not give any products, possibly because trifluoroacetic acid used in large excess as solvent, is too strong an acid. These initial results indicate the important role of acetic acid as a solvent with optimum $\mathrm{pKa}$ and, in some way, as an activator of the cyclization process.

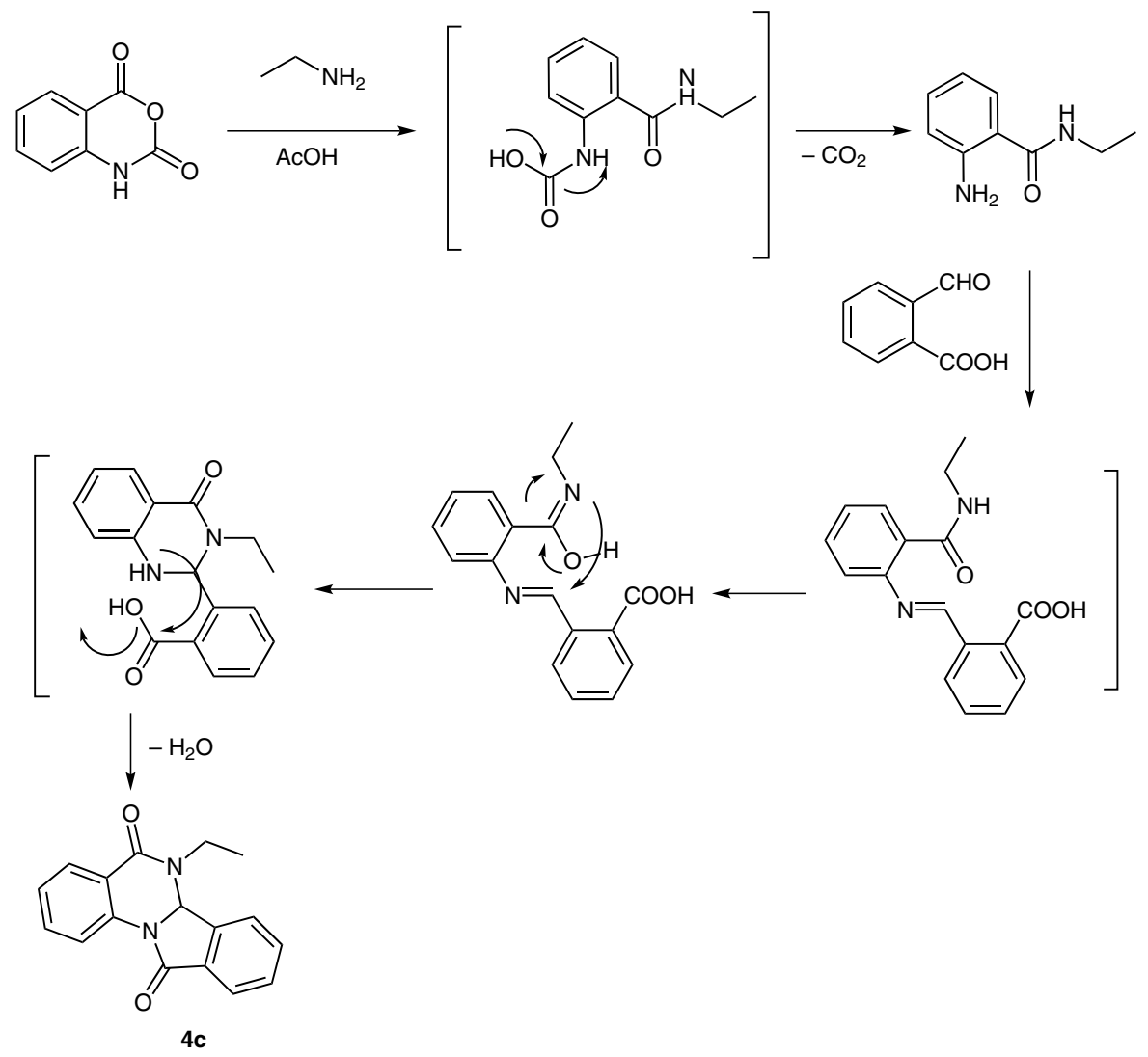

Scheme 2 Plausible mechanism for the formation of 6,6a-dihydroisoindolo[2,1-a]quinazoline-5,11-diones derivatives

Table 2 General Synthesis of 6,6a-Dihydroisoindolo[2,1-a]quinazoline-5,11-diones Derivatives from Suitable Precursors<smiles>O=c1[nH]c2ccccc2c(=O)o1</smiles>

1<smiles>N[18O]</smiles><smiles>O=Cc1ccccc1C(=O)O</smiles>

3

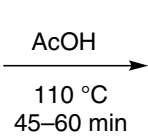
45-60 min<smiles>O=C1c2ccccc2N2C(=O)c3ccccc3C12</smiles>

\begin{tabular}{|c|c|c|c|c|}
\hline Entry & Amine 2 & Product 4 & Time (min) & Yield $(\%)^{\mathrm{a}}$ \\
\hline 1 & $\begin{array}{l}\mathrm{NH}_{4} \mathrm{OAc} \\
\mathbf{2 a}\end{array}$ & & 45 & 92 \\
\hline
\end{tabular}


Table 2 General Synthesis of 6,6a-Dihydroisoindolo[2,1-a]quinazoline-5,11-diones Derivatives from Suitable Precursors (continued)

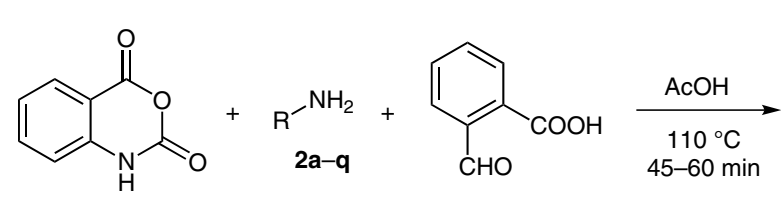

1

3<smiles>[R]N1C(=O)c2ccccc2N2C(=O)c3ccccc3C12</smiles>

\begin{tabular}{|c|c|c|c|c|}
\hline Entry & Amine 2 & Product 4 & Time (min) & Yield $(\%)^{\mathrm{a}}$ \\
\hline 2 & $\begin{array}{l}\mathrm{MeNH}_{2} \\
\text { 2b }\end{array}$ & & 50 & 92 \\
\hline
\end{tabular}

3

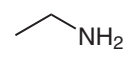

$2 \mathrm{c}$

4

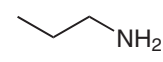

2d

5<smiles>NCCO</smiles>

$2 \mathrm{e}$<smiles>NCCCO</smiles>

$2 f$<smiles>NCCCC(=O)O</smiles>

$2 \mathrm{~g}$<smiles>CCN1C(=O)c2ccccc2N2C(=O)c3ccccc3C12</smiles>

$4 c$<smiles>CCCN1C(=O)c2ccccc2N2C(=O)c3ccccc3C12</smiles>

4d<smiles>O=C1c2ccccc2N2C(=O)c3ccccc3C2N1CCO</smiles>

$4 e$<smiles>O=C1c2ccccc2N2C(=O)c3ccccc3C2N1CCCO</smiles>

50

90

$4 f$<smiles>O=C(O)CCCN1C(=O)c2ccccc2N2C(=O)c3ccccc3C12</smiles>

55

89

$4 g$ 
Table 2 General Synthesis of 6,6a-Dihydroisoindolo[2,1-a]quinazoline-5,11-diones Derivatives from Suitable Precursors (continued)

$\underset{\mathrm{Ca}}{\stackrel{2 a-q}{+}}$

1

3<smiles>[R]N1C(=O)c2ccccc2N2C(=O)c3ccccc3C12</smiles>

Entry

Amine 2

Product 4

Time (min)

Yield (\%)

8

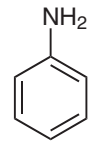

$2 h$<smiles>O=C1c2ccccc2N2C(=O)c3ccccc3C2N1c1ccccc1</smiles>

$4 h$<smiles>Cc1ccc(N2C(=O)c3ccccc3N3C(=O)c4ccccc4C23)cc1</smiles>

$4 i$<smiles>O=C1c2ccccc2N2C(=O)c3ccccc3C2N1c1ccc(Cl)cc1</smiles>

80

$4 j$<smiles>O=C1c2ccccc2N2C(=O)c3ccccc3C2N1c1ccc(O)cc1</smiles>

80<smiles>O=C1c2ccccc2N2C(=O)c3ccccc3C2N1c1cccc([N+](=O)[O-])c1</smiles>

60

82

41 
Table 2 General Synthesis of 6,6a-Dihydroisoindolo[2,1-a]quinazoline-5,11-diones Derivatives from Suitable Precursors (continued)

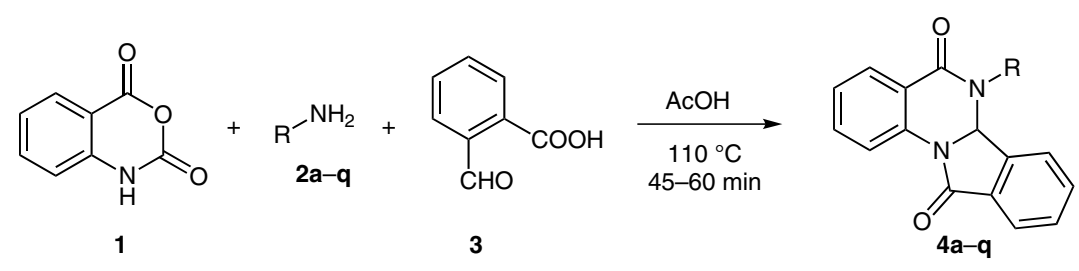

Entry Amine 2

14<smiles>COc1ccc(CN)cc1</smiles>

2n

15

16<smiles>NCc1ccc(F)cc1</smiles>

20<smiles>COc1ccc(CCN)cc1</smiles>

$2 p$

17<smiles>COc1ccc(CCN)cc1OC</smiles>

$2 q$<smiles>COc1ccc(CN2C(=O)c3ccccc3N3C(=O)c4ccccc4C23)cc1</smiles>

$4 n$<smiles>O=C1c2ccccc2N2C(=O)c3ccccc3C2N1Cc1ccc(F)cc1</smiles>

40<smiles>COc1ccc(CCN2C(=O)c3ccccc3N3C(=O)c4ccccc4C23)cc1</smiles>

$4 p$<smiles>COc1ccc(CCN2C(=O)c3ccccc3N3C(=O)c4ccccc4C23)cc1OC</smiles>

$4 q$

${ }^{a}$ Yields after recrystallization from chloroform-hexane (2:9).

The structural diversity of this reaction was further increased by using 2-aminobenzophenone (5b) instead of isatoic anhydride under similar reaction conditions, leading to the formation of new 5-phenylisoindolo[2,1a] quinazolin-11(6aH)-one derivatives (Table 3, compounds $\mathbf{6 a}-\mathbf{e})$.
According to Table 3, in all the cases the reactions were accomplished in relatively short reaction time and the products were obtained in good to excellent yields (more than $70 \%$ ). To determine the scope of this protocol, various aliphatic and aromatic amine derivatives were employed. However the reaction worked well with 2-amino- 


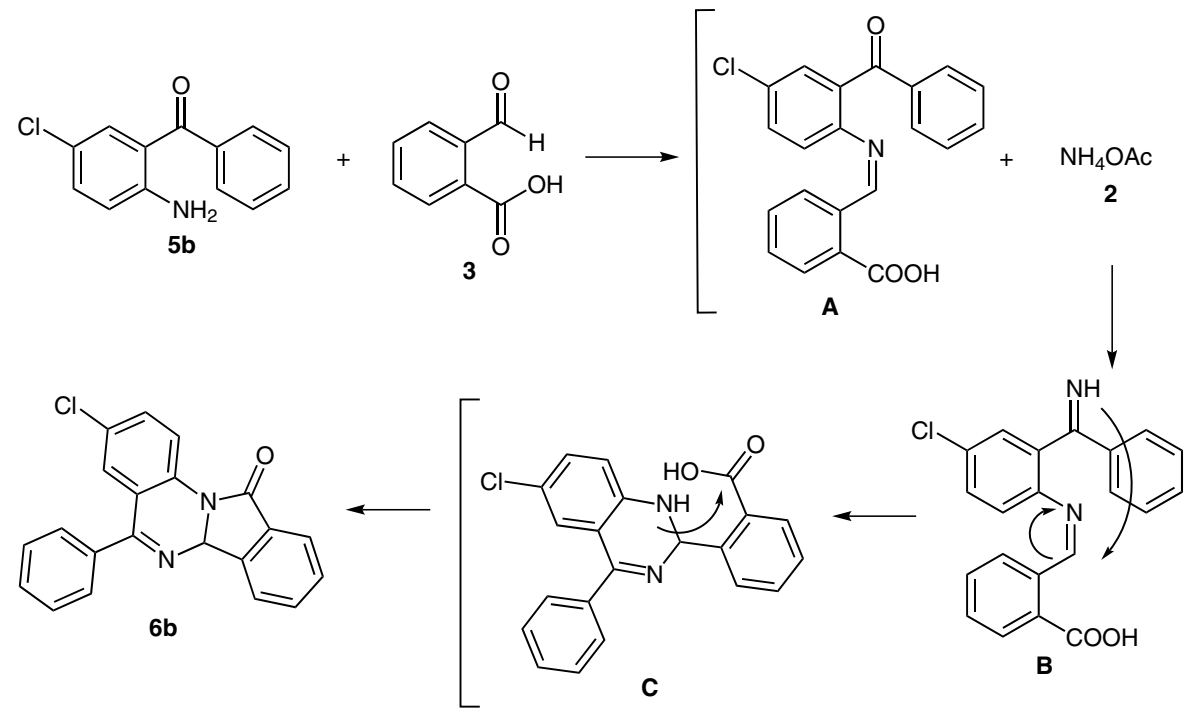

Scheme 3 Plausible mechanism for the formation of $\mathbf{6 b}$

benzophenone derivatives only. All compounds were characterized through ${ }^{1} \mathrm{H}$ NMR, ${ }^{13} \mathrm{C}$ NMR, HRMS and IR spectroscopic studies ${ }^{26}$ (please refer to the Supporting Information).

The proposed mechanism for the formation of the 5-phenylisoindolo[2,1-a]quinazolin-11(6aH)-ones is shown in Scheme $3 .^{23 a, 27,28}$ The reaction is expected to proceed via aldimine benzophenone intermediate $\mathbf{A}$ generated in situ from the reaction of $\mathbf{5} \mathbf{b}$ with 2 -formylbenzoic acid in acetic acid. The reaction of intermediate $\mathbf{A}$ with ammonium acetate results in the second intermediate imine $\mathbf{B}$ which subsequently participates in an intramolecular cyclization (C) followed by nucleophilic attack of the ring nitrogen on the carbonyl of the carboxylic acid leading to the formation of $6 \mathbf{b}$.
In summary, we have developed an alternative, catalystfree synthesis of 6,6a-dihydroisoindolo[2,1-a]quinazoline-5,11-diones and 5-phenylisoindolo[2,1-a]quinazolin$11(6 \mathrm{a} H)$-ones with excellent yields and short reaction times. Attractive features of this methodology are its versatility, the readily available starting materials needed, and the efficiency in creating a complex core in a single operation.

\section{Acknowledgment}

The authors are grateful to the Director, CDRI, Lucknow, India for constant encouragement, S.P. Singh for technical support, and SAIF for NMR, IR, and mass spectral data. G.R.P, R.P.D and A.S.R are thankful to CSIR, New Delhi, India for financial support. This is CDRI communication number 8354.

Table 3 Three-Component Synthesis of 5-Phenylisoindolo[2,1-a]quinazolin-11(6a $H)$-one Derivatives 6a-e in One Pot

3
3

${ }^{a}$ Yields are of the isolated product after purification by column chromatography. 
Supporting Information for this article is available online at http://www.thieme-connect.com/ejournals/toc/synlett.

\section{References and Notes}

(1) For quinazolines in medicinal chemistry, see: (a) Foster, B. A.; Coffrey, H. A.; Morin, M. J.; Rastinejad, F. Science 1999, 286, 2507. (b) Gundla, R.; Kazemi, R.; Sanam, R.; Muttineni, R.; Sarma, J. A. R. P.; Dayam, R.; Neamati, N. J. Med. Chem. 2008, 51, 3367. (c) Lüth, A.; Löwe, W. Eur. J. Med. Chem. 2008, 43, 1478. (d) Lewerenz, A.; Hentschel, S.; Vissiennon, Z.; Michael, S.; Nieber, K. Drug Dev. Res. 2003, 58, 420. (e) Doyle, L. A.; Ross, D. D. Oncogene 2003, 22, 7340. For quinazolinone, see: (f) Michael, J. P. Nat. Prod. Rep. 2004, 21, 650. (g) Mhaske, S. B.; Argade, N. P. Tetrahedron 2006, 62, 9787. For isoindolin-1-one, see: (h) Moreau, A.; Couture, A.; Deniau, E.; Grandclaudon, P. J. Org. Chem. 2004, 69, 4527. (i) Jagtap, P. G.; Southan, G. J.; Baloglu, E.; Ram, S.; Mabley, J. G.; Marton, A.; Salzman, A.; Szabó, C. Bioorg. Med. Chem. Lett. 2004, 14, 81. (j) Cappelli, A.; Gallelli, A.; Braile, C.; Anzini, M.; Vomero, S.; Mennuni, L.; Makovec, F.; Menziani, M. C.; De Benedetti, P. G.; Donati, A.; Giorgi, G. Bioorg. Med. Chem. 2002, 10, 2681.

(2) (a) Jiang, J. B.; Hesson, D.; Dusak, B.; Dexter, D.; Kang, G.; Hamel, E. J. Med. Chem. 1990, 33, 1721. (b) Cao, S. L.; Feng, Y. P.; Jiang, Y. Y.; Liu, S. Y.; Ding, G. Y.; Li, R. T. Bioorg. Med. Chem. Lett. 2005, 15, 1915.

(3) (a) Pendergast, W.; Johnson, J. V.; Dickerson, S. H.; Dev, I. K.; Duch, D. S.; Ferone, R.; Hall, W. R.; Humphreys, J.; Kelly, J. M.; Wilson, D. C. J. Med. Chem. 1993, 36, 2279. (b) Kung, P. P.; Casper, M. D.; Cook, K. L.; WilsonLingardo, L.; Risen, L. M.; Vickers, T. A.; Ranken, R.; Blyn, L. B.; Wyatt, J. R.; Cook, P. D. J. Med. Chem. 1999, 42, 4705.

(4) (a) Rorsch, F.; Buscato, E.; Deckmann, K.; Schneider, G.; Zsilavecz, M. S.; Geisslinger, G.; Proschak, E.; Grosch, S. J. Med. Chem. 2012, 55, 3792. (b) De Laszlo, S. E.; Quagliato, C. S.; Greenlee, W. J.; Patchett, A. A.; Chang, R. S. L.; Lotti, V. J.; Chen, T. B.; Scheck, S. A.; Faust, K. A. J. Med. Chem. 1993, 36, 3207.

(5) Chern, J. W.; Tao, P. L.; Wang, K. C.; Gutcait, A.; Liu, S. W.; Yen, M. H.; Chien, S. L.; Rong, J. K. J. Med. Chem. 1998, $41,3128$.

(6) Malamas, M. S.; Millen, J. J. Med. Chem. 1991, 34, 1492.

(7) Wolfe, J. F.; Rathman, T. L.; Sleevi, M. C.; Campbell, J. A.; Greenwood, T. D. J. Med. Chem. 1990, 33, 161.

(8) Mustazza, C.; Borioni, A.; Sestili, I.; Sbraccia, M.; Rodomonte, A.; Ferretti, R.; Giudice, M. R. D. Chem. Pharm. Bull. 2006, 54, 611.

(9) Bass, A. D.; Liu, M.; Dai, C.; Gray, K.; Nale, L.; Tevar, S.; Lee, S.; Liang, L.; Ponery, A.; Yaremko, B.; Smith, E.; Tang, H.; Sheth, P. R.; Siddiqui, M. A.; Hicklin, D. J.; Kirschmeier, P. Mol. Cancer Ther. 2010, 9, 2993.

(10) (a) Wood, K. W.; Bergnes, G. Annu. Rep. Med. Chem. 2004, 39, 173. (b) Bergnes, G.; Brejc, K.; Belmont, L. Curr. Top. Med. Chem. 2005, 5, 127.

(11) Audeval, B.; Bouchacourt, P.; Rondier, J. Gaz. Med. Fr. 1988, 95, 70 .

(12) Qin, J.; Rao, A.; Chen, X.; Zhu, X.; Liu, Z.; Huang, X.; Degrado, S.; Huang, Y.; Xiao, D.; Aslanian, R.; Cheewatrakoolpong, B.; Zhang, H.; Greenfeder, S.; Farley, C.; Cook, J.; Kurowski, S.; Li, Q.; Heek, M. V.; Chintala, M.; Wang, G.; Hsieh, Y.; Li, F.; Palani, A. ACS Med. Chem. Lett. 2011, 2, 171.

(13) Comins, D.; Schilling, S.; Zhang, Y. Org. Lett. 2005, 7, 95.
(14) (a) Chen, M.; He, M.; Zhou, X.; Huang, L.; Ruan, Y.; Huang, P. Tetrahedron 2005, 61, 1335. (b) Stuk, T. L.; Assink, B. K.; Bates, R. C.; Erdman, D. T.; Fedij, V.; Jennings, S. M.; Lassig, J. A.; Smith, R. J.; Smith, T. L. Org. Process Res. Dev. 2003, 7, 851. (c) Favor, D. A.; Powers, J. J.; White, A. D.; Fitzgerald, L. W.; Groppi, V.; Serpa, K. A. Bioorg. Med. Chem. Lett. 2010, 20, 5666. (d) Shirasaka, T.; Kunitake, T.; Tsuneyoshi, I. Brain Res. 2009, 1300, 105. (e) Augner, D.; Gerbino, D. C.; Slavov, N.; Neudorfl, J. M.; Schmalz, H. G. Org. Lett. 2011, 13, 5374.

(15) (a) Hyodo, S.; Fujita, K.; Kasuyaa, O.; Takahashia, I.; Uzawab, J.; Koshino, H. Tetrahedron 1995, 24, 6717. (b) Furumi, K.; Fujioka, T.; Fujii, H.; Okabe, H.; Nakano, Y.; Matsunaga, H.; Katano, M.; Mori, M.; Mihashi, K. Bioorg. Med. Chem. Lett. 1998, 8, 93.

(16) Oaksmith, J. M.; Ganem, B. Tetrahedron Lett. 2009, 26, 3497.

(17) Kortylewicz, Z. P.; Nearman, J.; Baranowska-Kortylewicz, J. J. Med. Chem. 2009, 16, 5124.

(18) Vasilevsky, S. F.; Govdi, A. I.; Sorokina, I. V.; Tolstikova, T. G.; Baev, D. S.; Tolstikov, G. A.; Mamatuyk, V. I.; Alabugin, I. V. Bioorg. Med. Chem. Lett. 2011, 21, 62.

(19) (a) Breiner, B.; Schlatterer, J. C.; Kovalenko, S. V.; Greenbaum, N. L.; Alabugin, I. V. Proc. Natl. Acad. Sci. U.S.A. 2007, 104, 13016. (b) Breiner, B.; Schlatterer, J. C.; Kovalenko, S. V.; Greenbaum, N. L.; Alabugin, I. V. Angew. Chem. Int. Ed. 2006, 45, 3666. (c) Kovalenko, S. V.; Alabugin, I. V. Chem. Commun. 2005, 1444. (d) Yang, W.Y.; Breiner, B.; Kovalenko, S. V.; Ben, C.; Singh, M.; Le Grand, S. N.; Sang, Q.-X.; Strouse, G. F.; Copland, J. A.; Alabugin, I. V. J. Am. Chem. Soc. 2009, 131, 11458. (e) Yang, W.-Y.; Marrone, S. A.; Minors, N.; Zorio, D. A. R.; Alabugin, I. V. Beilstein J. Org. Chem. 2011, 7, 813.

(20) (a) Foye, W. O. Principidi Chemico Farmaceutica; Piccin: Padora (Italy), 1991, 416. (b) Andreani, L. L.; Lapi, E. Bull. Chim. Farm. 1960, 99, 583. (c) Bonsignore, L.; Loy, G.; Secci, D.; Calignano, A. Eur. J. Med. Chem. 1993, 28, 517.

(21) Ellis, G. P. In The Chemistry of Heterocyclic Compounds: Chromenes, Chromanes and Chromones; Weissberger, A.; Taylor, E. C., Eds.; John Wiley: New York (NY), 1977, Chap. II 11.

(22) Arnesto, D.; Horspool, W. M.; Martin, N.; Ramos, A.; Seaone, C. J. Org. Chem. 1989, 54, 3069.

(23) (a) Siva Kumar, K.; Mahesh Kumar, P.; Anil Kumar, K.; Sreenivasulu, M.; Ahamed, A. J.; Rambabu, D.; Rama Krishna, G.; Malla Reddy, C.; Ravikumar, K.; Shivakumar, K.; Krishna Priya, K.; Kishore, V. L. P.; Pal, M. Chem. Commun. 2011, 47, 5010. (b) Bunce, R. A.; Nammalwar, B. J. Heterocycl. Chem. 2011, 48, 991.

(24) (a) Sashidhara, K. V.; Palnati, G. R.; Avula, S. R.; Kumar, A. Synlett 2012, 23, 611. (b) Sashidhara, K. V.; Avula, S. R.; Singh, L. R.; Palnati, G. R. Tetrahedron Lett. 2012, 53, 4880. (c) Sashidhara, K. V.; Kumar, A.; Dodda, R. P.; Kumar, B. Tetrahedron Lett. 2012, 53, 3281. (d) Sashidhara, K. V.; Kumar, M.; Kumar, A. Tetrahedron Lett. 2012, 53, 2355. (e) Sashidhara, K. V.; Kumar, A.; Agarwal, S.; Kumar, M.; Kumar, M.; Sridhar, B. Adv. Synth. Catal. 2012, 354, 1129. (f) Sashidhara, K. V.; Kumar, A.; Rao, K. B. Tetrahedron Lett. 2011, 52, 5659.

(25) Representative Procedure for the Synthesis of 6-Ethyl6,6a-dihydroisoindolo[2,1-a]quinazoline-5,11-dione: Isatoic anhydride $(1.0 \mathrm{mmol})$ was added to a $50-\mathrm{mL}$ roundbottom flask containing AcOH $(10 \mathrm{~mL})$. Ethylamine $(1.1$ $\mathrm{mmol})$ and 2-formylbenzoic acid $(1.0 \mathrm{mmol})$ were added and the reaction mixture was heated for $45 \mathrm{~min}$ at $110^{\circ} \mathrm{C}$ (initially effervescence was observed due to the generation of $\mathrm{CO}_{2}$ gas). After completion of the reaction (TLC), the 
reaction mixture was cooled to r.t. and poured into cold $\mathrm{H}_{2} \mathrm{O}$ to precipitate the crude product which was filtered off. The crude product was recrystallized from $\mathrm{CHCl}_{3}$-hexane $(2: 8)$ to give the pure product $4 \mathbf{c}$ as a white solid; yield: $91 \%$; $\mathrm{mp}$ $156-158^{\circ} \mathrm{C}$. IR (KBr): 3016, 2854, 1718, 1655, 1487, 1064 $\mathrm{cm}^{-1} .{ }^{1} \mathrm{H}$ NMR $\left(300 \mathrm{MHz}, \mathrm{CDCl}_{3}\right): \delta=8.09-8.16(\mathrm{~m}, 2 \mathrm{H})$, $8.05(\mathrm{~d}, J=6.9 \mathrm{~Hz}, 1 \mathrm{H}), 7.60-7.80(\mathrm{~m}, 4 \mathrm{H}), 7.31-7.35(\mathrm{~m}$, $1 \mathrm{H}), 6.25(\mathrm{~s}, 1 \mathrm{H}), 3.98-4.10(\mathrm{~m}, 1 \mathrm{H}), 3.69-3.81(\mathrm{~m}, 1 \mathrm{H})$, $1.23(\mathrm{t}, J=7.0 \mathrm{~Hz}, 3 \mathrm{H}) \cdot{ }^{13} \mathrm{C} \mathrm{NMR}\left(75 \mathrm{MHz}, \mathrm{CDCl}_{3}\right): \delta=$ 164.8, 163.5, 138.1, 136.6, 133.3, 132.8, 132.7, 130.5, 128.9, 125.1, 125.1, 125.0, 120.5, 120.1, 70.2, 37.7, 13.3. ESI-MS: $m / z=279[\mathrm{M}+\mathrm{H}]^{+}$.

(26) General Procedure for the Preparation of 3-Chloro-5phenylisoindolo[2,1-a]quinazolin-11(6aH)-one (6b): 2Amino-5-chlorobenzophenone (1.0 mmol) was added to a $50-\mathrm{mL}$ round-bottom flask containing $\mathrm{AcOH}(10 \mathrm{~mL})$. Ammonium acetate (excess) and 2-formylbenzoic acid (1.0 $\mathrm{mmol}$ ) were then added and the reaction mixture was heated for $45 \mathrm{~min}$ at $110^{\circ} \mathrm{C}$. After completion of the reaction (TLC), the reaction mixture was cooled to r.t. and poured into cold $\mathrm{H}_{2} \mathrm{O}$ to precipitate the crude product which was filtered off. The crude product was purified by column chromatography $\left(\mathrm{CH}_{2} \mathrm{Cl}_{2}\right.$-hexane, 5:5). The product $\mathbf{6 b}$ was obtained as a pale green solid; yield: $80 \%$; $\mathrm{mp} 216-218^{\circ} \mathrm{C}$. IR (KBr): 2922, 1646, $1218 \mathrm{~cm}^{-1} .{ }^{1} \mathrm{H}$ NMR $(300 \mathrm{MHz}$, $\left.\mathrm{CDCl}_{3}\right): \delta=8.07(\mathrm{~d}, J=7.4 \mathrm{~Hz}, 1 \mathrm{H}), 7.80(\mathrm{~d}, J=7.3 \mathrm{~Hz}, 1$ H), 7.69-7.73 (m, 1 H), 7.60-7.65 (m, $1 \mathrm{H}), 7.48(\mathrm{~d}, J=8.4$ $\mathrm{Hz}, 1 \mathrm{H}), 7.25-7.30(\mathrm{~m}, 6 \mathrm{H}), 7.05(\mathrm{~d}, J=2.2 \mathrm{~Hz}, 1 \mathrm{H}), 6.27$ $(\mathrm{s}, 1 \mathrm{H}) .{ }^{13} \mathrm{C} \mathrm{NMR}\left(50 \mathrm{MHz}, \mathrm{CDCl}_{3}\right): \delta=166.5,149.3$, 141.7, 138.7, 134.5, 133.3, 133.2, 132.4, 130.4, 129.1, 129.1, 128.5, 128.1, 127.7, 127.4, 123.6, 122.4, 55.7. HRMS (ESI): $m / z[\mathrm{M}+\mathrm{H}]$ calcd for $\mathrm{C}_{21} \mathrm{H}_{13} \mathrm{~N}_{2} \mathrm{ClO}: 345.0795$; found: 345.0785 .

(27) (a) Hosseini-Zare, M. S.; Mahdavi, M.; Saeedi, M.; Asadi, M.; Javanshir, S.; Shafiee, A.; Foroumadi, A. Tetrahedron Lett. 2012, 53, 3448. (b) Kumar, K. S.; Kumar, P. M.; Rao, V. S.; Jafar, A. A.; Meda, C. L. T.; Kapavarapu, R.; Parsa, K. V. L.; Pal, M. Org. Biomol. Chem. 2012, 10, 3098.

(28) Panja, S. K.; Dwivedi, N.; Saha, S. Tetrahedron Lett. 2012 , 53,6176 . 